\title{
Initial Versus Final Diagnosis in Patients Who Presented to the Emergency Department Without Trauma: A Prospective Cohort Study
}

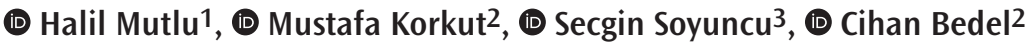 \\ ${ }^{1}$ Clinic of Emergency Medicine, University of Health Sciences Turkey, Gülhane Training and Research Hospital, İstanbul, Turkey \\ ${ }^{2}$ Clinic of Emergency Medicine, University of Health Sciences Turkey, Antalya Training and Research Hospital, Antalya, Turkey \\ ${ }^{3}$ Department of Emergency Medicine, Akdeniz University Faculty of Medicine, Antalya, Turkey
}

\begin{abstract}
Aim: In the emergency department (ED), some patients are discharged after their initial diagnosis and treatment, whereas others are hospitalized for treatment and/or further diagnostic examination. The ED physician usually does not receive feedback regarding diagnostic accuracy, treatment effectiveness, or morbidity or mortality of the patient. In this study, patients in the ED without trauma were followed up to obtain their data.

Materials and Methods: This prospective cohort study includes all patients without trauma who are admitted to the ED of a tertiary hospital during the two-month time frame and were hospitalized in various clinics. Data recorded for each patient are the following: demographic information, vital signs, diagnosis upon admission, diagnosis after hospitalization, length of stay, mortality, and complications.

Results: A total of 740 patients that met the inclusion criteria participated in this study. The mean age was 54 years, wherein 398 patients (53.8\%) were male. The initial diagnosis of 22 patients (2.9\%) changed after further examinations. The mean age of these patients were 42 years, and 11 patients were male (50\%). Emergency invasive intervention was significantly more common among patients with changed diagnosis (cDx) $(40.9 \%$ vs $4.1 \%, p<0.001)$. Consequently, the incidence of complications was higher in cDx patients $(31.8 \%$ vs $10.8 \%$, $p=0.01)$.

Conclusion: Majority of patients hospitalized from the ED were treated according to their initial diagnosis until the initial department of hospitalization. We conclude that ED functions at an adequate accuracy despite their high workload.
\end{abstract}

Keywords: Emergency department, hospitalization, non-traumatic complications, mortality, diagnosis

\section{Introduction}

Emergency departments (EDs) are designed to provide continuous medical care to ensure that fast decisions are made in order to prevent patient death and disability. People come to EDs with a great variety of diseases, which translate in to differences in diagnosis and treatment of physical and behavioral problems.

Some patients are discharged following initial diagnosis and treatment, whereas others are hospitalized for treatment and/ or further diagnostic examination (1-3). As the ED physician does not follow up with treatment after hospitalization, they usually do not receive feedback regarding the accuracy of their diagnosis, the effectiveness of the treatment, or the morbidity/ mortality of the patient. The literature review revealed several studies concerning changed and missed diagnoses among trauma patients (3-6). However, there are only a few articles that concern this issue. Therefore, we aimed to evaluate the demographic characteristics, complications and the initial and final diagnoses of non-traumatic patients that were hospitalized from ED.

\section{Materials and Methods}

This prospective cohort study was approved by the local ethics committee (decision no: 521, date:10.12.2014), and was conducted in a tertiary care university hospital ED in accordance with the 
Helsinki Declaration Principles. A total of 16,672 patients were treated in the ED during the two-month period, and 740 (4.4\%) of these patients were hospitalized (Figure 1). The inclusion criteria were: (a) patients under 18 years old, (b) initial admission to ED, (c) hospitalization. The exclusion criteria were: (a) traumatic injury, (b) hospitalization in a different medical center due to hospital reaching capacity. A form was created for the followup and treatment of hospitalized patients, which was filled by the ED physician during treatment. Diagnostic evaluation was recorded by specialist doctors and residents with $>3$ years' experience. The following data were recorded for each patient: demographic information, vital signs, triage levels, diagnosis at the time of admission, diagnosis after hospitalization, length of stay, mortality and complications during treatment. The triage category of the patients was made (green, yellow, red, black) and the patients were grouped. A change in the initial diagnosis at follow-up was noted. Patients with and without diagnostic changes were compared in terms of parameters. However, the clinical diagnosis may differ from the initial diagnosis, and therefore the main diagnosis was targeted in these cases. The primary outcome of the present study was to determine the initial and final diagnoses of non-traumatic patients that were hospitalized from ED. The second outcome was to investigate the changes in diagnosis and the outcome of these patients.

\section{Statistical Analysis}

The data were analyzed using SPSS version 20.0. Demographic data were assessed by descriptive tests, and expressed as percentages, mean \pm standard deviation, or median and

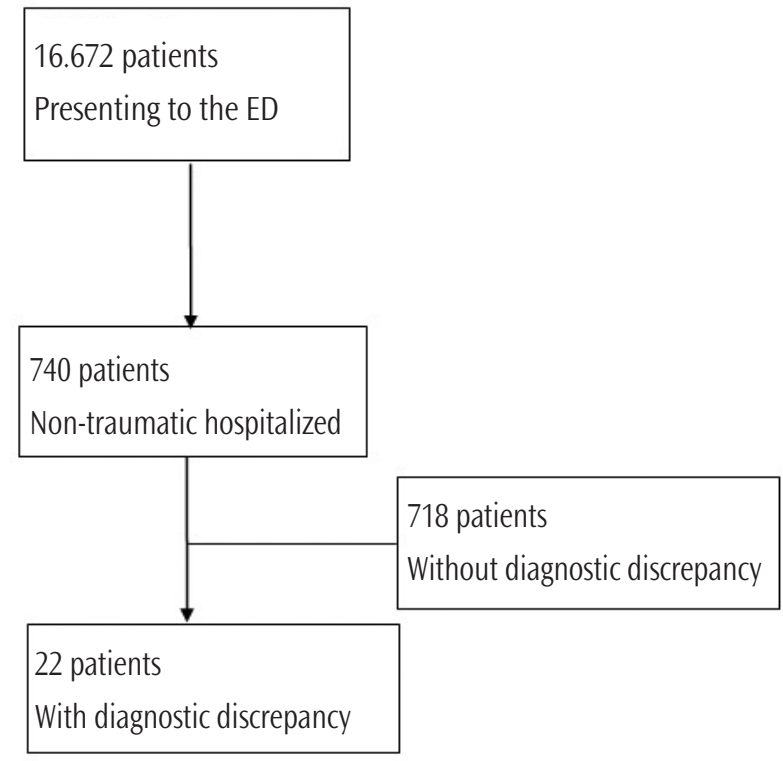

Figure 1. Patient flow chart

ED: Emergency depatment interquartile range. Chi-square test was used for the comparison of categorical variables. All hypotheses were bi-directional, and significance level was set at 0.05 .

\section{Results}

A total of 740 subjects were included in the study. Mean age was 54 years, 398 patients (53.8\%) were male. Median GCS at the time of admission 14.5, and GCS was below 8 for 11 patients (1.4\%). Mean hospital length of stay was $7.36 \pm 8.55$ days, and there were 40 hospital deaths (5.4\%). The general characteristics of the subjects are presented in Table 1.

The initial diagnosis of 22 patients (2.9\%) was changed after further examinations (CDx). Mean age of $\mathrm{cDx}$ patients was 42 , and

\begin{tabular}{|c|c|}
\hline Included patients & 740 \\
\hline Age years (P25-75) & $54(38-68.75)$ \\
\hline Male sex, n (\%) & $398(53.8 \%)$ \\
\hline GCS $<8$ at presentation, $\mathrm{n}(\%)$ & $11(1.4 \%)$ \\
\hline \multicolumn{2}{|l|}{ Vital signs, initial } \\
\hline Systolic blood pressure mmHg, (P25-75) & $126(110-146)$ \\
\hline Diastolic blood pressure mmHg, (P25-75) & $73(60-85)$ \\
\hline Heart rate beats per minute, (P25-75) & $96(81-125)$ \\
\hline Temperature ${ }^{\circ} \mathrm{C},(\mathrm{P} 25-75)$ & $36.2(36-36.8)$ \\
\hline Oxygen saturation, \% (P25-75) & $98(95-99)$ \\
\hline Concomitant disease, n (\%) & $467(63.1 \%)$ \\
\hline \multicolumn{2}{|l|}{ Circumstantial factors } \\
\hline Waiting time in ED, (min) & $188.26 \pm 168.76$ \\
\hline \multicolumn{2}{|l|}{ Time of arrival } \\
\hline Daytime (08:00-16:00) & $304(41.1 \%)$ \\
\hline Evening (16:00-00:00) & $302(40.8 \%)$ \\
\hline Night time (00:00-08:00) & $134(18.1 \%)$ \\
\hline Emergency intervention & $39(5.3 \%)$ \\
\hline \multicolumn{2}{|l|}{ Triage category, $\mathbf{n}(\%)$} \\
\hline 1 & $38(5.1 \%)$ \\
\hline 2 & $202(27.3 \%)$ \\
\hline 3 & $483(65.3 \%)$ \\
\hline 4 & $17(2.3 \%)$ \\
\hline Primary ICU admission & $106(14.3 \%)$ \\
\hline \multicolumn{2}{|l|}{ Medical outcomes } \\
\hline Complications & $85(11.4 \%)$ \\
\hline In-hospital mortality, n (\%) & $40(5.4 \%)$ \\
\hline Length of hospital stay (day) & $7.36 \pm 8.55$ \\
\hline \multicolumn{2}{|c|}{$\begin{array}{l}\text { GCS: Glasgow Coma Scale, ED: Emergency department, ICU: Intensive care unit, } \\
\text { Number }\end{array}$} \\
\hline
\end{tabular}


11 were male (50\%). Mean length of ED stay was $166.18 \pm 87.19$ minutes, mean systolic blood pressure was $123.5 \mathrm{mmHg}$, mean diastolic blood pressure was $73.5 \mathrm{mmHg}$, and mean length of stay was $7.33 \pm 8.62$ days. Emergency invasive intervention was significantly more common among cDx patients $(40.9 \%$ vs $4.1 \%$, $\mathrm{p}<0.001)$. Consequently, the incidence of complications was higher in $c D x$ patients ( $31.8 \%$ vs $10.8 \%, p=0.01$ ). There was no significant difference between the groups in terms of other parameters $(p>0.05)$. The comparison of demographic and etiological data is presented in Table 2. For cDx patients, initial ED diagnosis, diagnosis at the time of discharge, and the clinics that the patients were treated in are presented in Table 3.

\section{Discussion}

Majority of ED patients are discharged following initial diagnosis and treatment, whereas others are hospitalized for treatment and/or further diagnostic examination. Since the ED physician does not follow up with the patient after hospitalization, they usually do not receive feedback regarding the accuracy of their diagnosis, the effectiveness of the treatment, or the morbidity/ mortality of the patient. The literature review revealed several studies concerning changed and missed diagnoses among trauma patients (4-8). Therefore, we chose to study non-traumatic patients. We aimed to evaluate the demographic characteristics, complications and the initial/final diagnoses of non-traumatic patients that were hospitalized from ED.

A total of 16,672 patients were treated in the ED during the twomonth period. This study concerns non-traumatic patients that were treated in an adult ED, thus it does not include patients aged below 16 years. A total of 740 patients were included in the study, overall hospitalization rate was $4.4 \%$. The hospitalization rate of the same hospital was $12.5 \%$ in previous years (9). Akpinar et al. (10) found this rate to be $12.8 \%$. Another study conducted among patients hospitalized in the intensive care unit from the ED of a university hospital in the same country found average duration of ED stay to be 300 minutes $(11,12)$. In our study, average stay in ED was $188.26 \pm 168.76$ minutes. This relatively short average length of stay might be due to the low number of

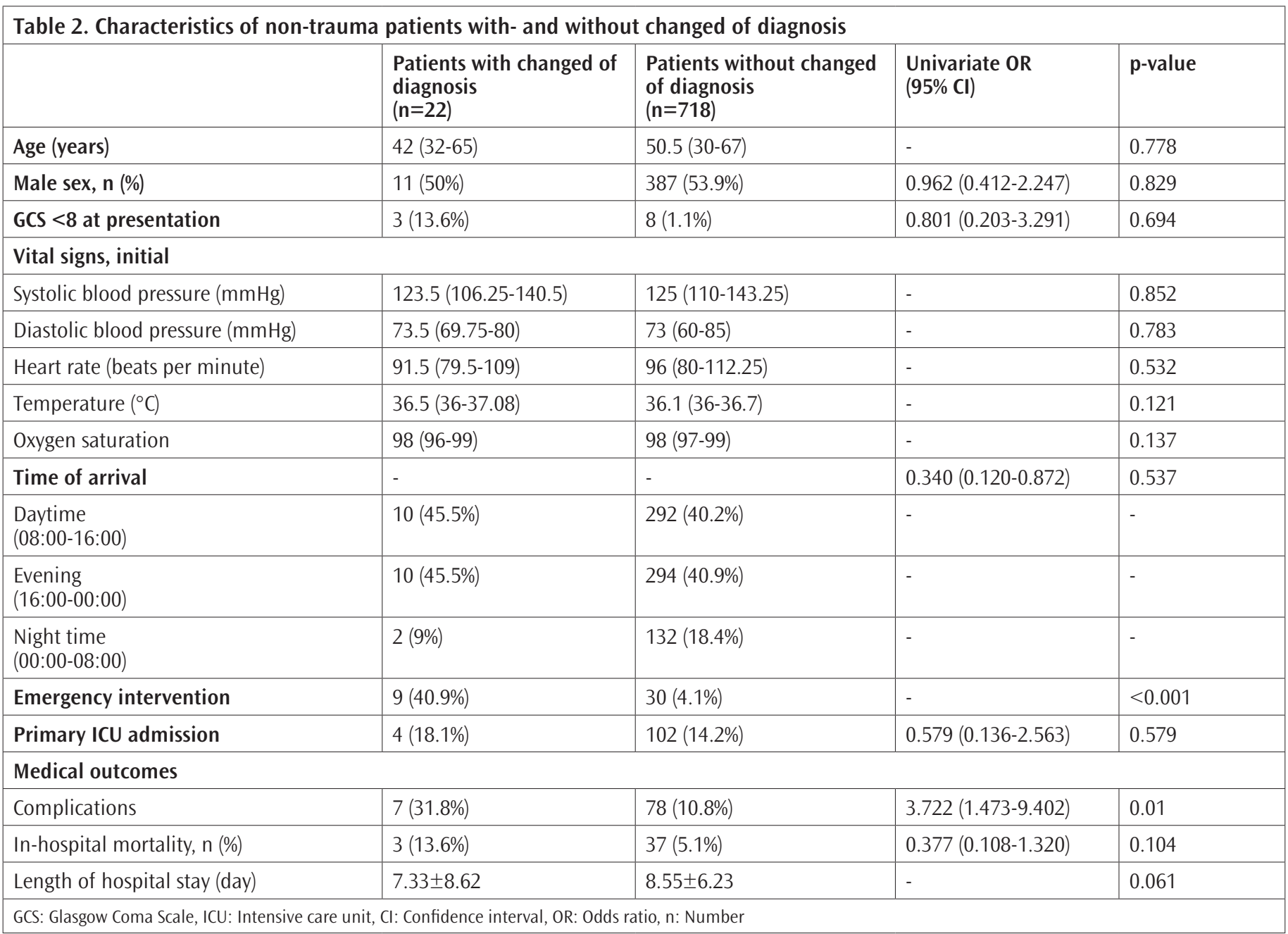




\begin{tabular}{|c|c|c|c|}
\hline First diagnosis in ED & Last diagnosis & Mortality & Clinics \\
\hline ACS & Brain tumour + pulmonary mass & No & Cardiology \\
\hline ACS & Intestinal perforation & Yes & Cardiology \\
\hline Cerebrovascular disease & Metabolic disorder & No & Neurology \\
\hline Cerebrovascular disease & Hypertensive encephalopathy & No & Neurology \\
\hline Bradycardia & Exacerbations of COPD & No & Cardiology \\
\hline Cholecystitis & Pneumatosis carcionmatosa & No & General surgery \\
\hline Choledocholithiasis & Liver cancer & Yes & Internal medicine \\
\hline Acute renal failure & Acute adrenal insufficiency & No & Internal medicine \\
\hline Abortion & Ectopic pregnancy & No & Obstetrics and gynaecology \\
\hline Submandibular abscess & Brain tumour & No & Ear nose throat \\
\hline Anemia etiology & Mantle cell lymphoma & No & Internal medicine \\
\hline Etiology of fever & Infective arthritis & No & Infectious diseases \\
\hline Asthma attack & Bronchiectasis & No & Chest diseases \\
\hline Anemia etiology & Hemarthroses & No & Infectious diseases \\
\hline Pulmonary edema & Breast cancer & Yes & Cardiology \\
\hline Transverse myelitis & Multiple sclerosis & No & Neurology \\
\hline Lumbar disc herniation & Spinal tumour & No & Neurosurgery \\
\hline Exacerbations of COPD & Pulmonary mass & No & Chest diseases \\
\hline Renal abscess & Renal mass & No & Urology \\
\hline Nonspecific abdominal pain & Portal vein thrombosis & No & Internal medicine \\
\hline Pyelonephritis & Hemorrhagic ovarian cyst & No & Internal medicine \\
\hline Acute appendicitis & Inflammatory bowel disease & No & General surgery \\
\hline
\end{tabular}

intensive care unit hospitalizations. The most important factor affecting duration of stay in ED is specialist consultations (13-16). Increasing age and comorbidities require the inclusion of more clinical departments in the treatment. In addition, comorbidities that concern different clinics bring about the requirement of choice between these clinics for hospitalization, leading to prolonged length of stay. In our study, there were 38 (5.1\%) Level 1 and 483 (65.3\%) Level 3 triage patients, thus, the majority of the subjects were Level 3 triage patients. This factor also contributes to the relatively short average length of ED stay.

The initial diagnoses of 22 subjects (2.9\%) were different that the diagnosis at discharge (cDx). Giannakopoulos et al. (4) conducted a similar study, in which they found this rate to be $8.2 \%$, whereas Chen et al. (17) found it to be $12.1 \%$. They also found that $89.6 \%$ of $\mathrm{CDx}$ patients had life-threatening conditions. In our study, 11 (50\%) of cDx patients were male, and the mean age was 42 years. One study found the mean age of CDx patients to be 38.6, and that $69.5 \%$ were male (17). Another study found that age and gender were not significant factors in diagnosis change (4).
In our study, the mean length of ED stay (from admission until hospitalization) of cDx patients was $166.18 \pm 87.19$ minute, and mean length of stay was $7.33 \pm 8.62$ days. Another study of 976 patients, found the mean length of ED stay and mean length of stay to be 18.5 minutes and 4.3 days, respectively. We believe that the difference in length of ED stay is due to the difference in patients' comorbidities and hospital policies. In our study, most cDx patients had applied between 08:00-16:00 $(n=10$, 45.5\%). However, Chen et al. (17) reported that the most common application time for CDx patients was between 16:00-24:00 (41.5\%). In both studies, the smallest number of applications was between 00:00-08:00, as consistent with the literature (18).

Emergency invasive intervention was significantly more common among cDx patients ( $40.9 \%$ vs $4.1 \%, p<0.001)$. Also, the incidence of complications was higher in cDx patients ( $31.8 \%$ vs $10.8 \%$, $p=0.01$ ). One study found this rate to be $23.9 \%$ (17); however, the shorter length of ED stay in this study may have led to the comparatively low rate. Another study found the rate of 
complications to be $5.9 \%$ in cDx patients; however, unlike our study, this difference was not significant (17).

The symptom that is the most difficult to distinguish is abdominal and chest pain. Therefore, patients with epigastric pain should be examined for acute coronary syndrome (ACS) (19). In our study, one patient was admitted to the cardiology clinic with potential ACS; however, further examinations revealed intestinal perforation, and the patient died during surgical intervention.

\section{Study Limitations}

The limitations of our study are as follows: (a) the limited time frame and the relatively small sample size, (b) exclusion of physical examination findings and symptoms, (c) differences between the diagnosis and treatment methods among clinicians, despite being specialists or senior residents, (d) different treatment and protocols after hospitalization, and not standardizing the physicians making the final diagnosis. The scarcity of relevant literature requires multicenter and prospective studies.

\section{Conclusion}

EDs provide intensive medical care, and make up a significant source of hospitalizations. In our study, majority of patients that were hospitalized from the ED were treated with their initial diagnosis and in the initial department of hospitalization. We conclude that the ED performs physical examination, imaging, laboratory and consultation functions at an adequate accuracy despite their high workload.

\section{Ethics}

Ethics Committee Approval: Prior to implementation, this study's protocol was approved by Akdeniz University Ethics Committee (decision no: 521, date: 10/12/2014).

Informed Consent: An informed consent form was obtained from each patient.

Peer-review: Externally peer-reviewed.

\section{Authorship Contributions}

Surgical and Medical Practices: H.M., S.S., Concept: H.M., M.K., S.S., C.B., Design: H.M., M.K., S.S., C.B., Data Collection or Processing: H.M., M.K., S.S., Analysis or Interpretation: H.M., S.S., C.B., Literature Search: H.M., M.K., S.S., C.B., Writing: H.M., M.K., S.S., C.B.

Conflict of Interest: No conflict of interest was declared by the authors.

Financial Disclosure: The authors declared that this study received no financial support.

\section{References}

1. Ukkonen M, Jämsen E, Zeitlin R, Pauniaho SL. Emergency department visits in older patients: a population-based survey. BMC Emerg Med. 2019;19:20.

2. Moe J, Kirkland SW, Rawe E, Ospina MB, Vandermeer B, Campbell S, et al. Effectiveness of interventions to decrease emergency department visits by adult frequent users: a systematic review. Acad Emerg Med. 2017;24:40-52.

3. Moonen PJ, Mercelina L, Boer W, Fret T. Diagnostic error in the Emergency Department: follow up of patients with minor trauma in the outpatient clinic. Scand J Trauma Resusc Emerg Med. 2017;25:13.

4. Giannakopoulos G, Saltzherr T, Beenen L, Reitsma J, Bloemers F, Goslings JC, et al. Missed injuries during the initial assessment in a cohort of 1124 level-1 trauma patients. Injury. 2012;43:1517-21.

5. Ferguson B, Geralds J, Petrey J, Huecker M. Malpractice in Emergency Medicine-a review of risk and mitigation practices for the emergency medicine provider. J Emerg Med. 2018;55:659-65.

6. Fernholm R, Pukk Härenstam K, Wachtler C, Nilsson GH, Holzmann MJ, Carlsson AC. Diagnostic errors reported in primary healthcare and emergency departments: A retrospective and descriptive cohort study of 4830 reported cases of preventable harm in Sweden. Eur J Gen Pract. 2019;25:128-35.

7. Okafor N, Payne VL, Chathampally Y, Miller S, Doshi P, Singh H. Using voluntary reports from physicians to learn from diagnostic errors in emergency medicine. Emerg Med J. 2016;33:245-52.

8. Weinberger SE. Providing High-value, cost-conscious care: a critical seventh general competency for physicians. Ann Intern Med. 2011;155:386-88.

9. Kılıçaslan I, Bozan H, Oktay C, Göksu E. Demographic properties of patients presenting to the emergency department in Turkey. Turk J Emerg Med. 2005;5:5-13.

10. Akpinar O, Turkdogan KA, Kapci M, Duman A. Use of emergency department by elderly patients. J Clin Anal Med. 2015;6:310-4.

11. Sert PC, Durak VA, Ozdemir F, Armağan E. Determination of the appropriate emergency department design according to patient characteristics. J Uludag Uni Med Fac. 2017;43:17-21

12. Dikme O, Atilla R, Oray NC, Saracoglu G, Girgin MC, Akyol PY. Dokuz Eylul Triage System: A five category triage algorithm, reliability and validity study. Anatolian J Emerg Med. 2019:2:1-7.

13. Collins A, Barnicot K, Sen P. A systematic review and meta-analysis of personality disorder prevalence and patient outcomes in emergency departments. J Pers Disord. 2018:1-24.

14. Warren MB, Campbell RL, Nestler DM, Pasupathy KS, Lohse CM, Koch KA, et al. Prolonged length of stay in ED psychiatric patients: a multivariable predictive model. The Am J Emerg Med. 2016;34:133-9.

15. Hoffmann JA, Stack AM, Monuteaux MC, Levin R, Lee LK. Factors associated with boarding and length of stay for pediatric mental health emergency visits. Am J Emerg Med. 2018;11:31003-9.

16. Shenvi C, Wilson MP, Aldai A, Pepper D, Gerardi M. A research agenda for the assessment and management of acute behavioral changes in elderly emergency department patients. West J Emerg Med. 2019;20:393.

17. Chen CW, Chu CM, Yu WY, Lou YT, Lin MR. Incidence rate and risk factors of missed injuries in major trauma patients. Accid Anal Prev. 2011;43:823-8.

18. Gürsoy V, Coșkun BN, Öksüz MF, Tufan AN, Ermurat S, Kutlu N, et al. Rheumatology consultation in the emergency department: Annual results. J Turk Soc Rheumatol. 2014;6:8-12.

19. Fang SL, Deng M, Peng YN, Gao C, Zhao Q, Liu J, et al. Gastric volvulus presenting as an acute coronary syndrome with long-lasting electrocardiographic changes. J Geriatr Cardiol. 2019;16:309-12. 